\title{
Dynamics of Non-Interacting Fluids in Kantowski-Sachs Universe with a Cosmological Constant
}

\author{
S. M. Borikar
}

\begin{abstract}
In this present study we have investigated Kantowski-Sachs universe filled with perfect fluid and radiation with a cosmological constant. To get determinate solution, it is assumed that the shear scalar is proportional to $\mathrm{s}$ scalar of expansion hear scalar, which yields the relation between metric potentials as $R=A S^{n}$. The cosmological parameters of models are also discussed.
\end{abstract}

Index Terms - Perfect fluid, Radiation, cosmological constant, Kantowski-Sachs space-time

\section{INTRODUCTION}

The spatially homogeneous and anisotropic Kantowski-Sachs model has astro-physically important as it represent early era in cosmology. The recent observations indicate that the universe is expanding and on large scale it is homogeneous and isotropic (Knop et.al (2003), Gasprini (2003), Riess et.al. (2004)) and is in accelerating phase. Roy Choudhari (1979) have studied the solutions of homogeneous space-time belongs to either Bianchi types or Kantowski-Sachs in general relativity.

Kantowski-Sachs models have been studied by Weber (1984, 85), Lorcaz (1983), Gron (1986), Matravers (1988), Krori et.al.(1995), Li \& Hao (2003). Sing and Agrawal have analyzed Kantowski-Sachs model in Seaz Ballester (1985) and scalar tensor theory (1991). Pradhan and Yadav (2002) have obtained the solutions for Kantowski-Sachs model with variable $\mathrm{G}$ and . Anisotropic dark energy model has been investigated and studied by Adhav et.al. (2011) in Kantowski-Sachs space-tine.

To understand the early and present stages of the universe, the FRW models are considered as standard cosmological models. Many authors investigated two fluid FRW models (Davidson 1962; McIntosh 1968). Two fluid models where one fluid is the radiation corresponding to the observed cosmic background radiation, while the matter content of the universe is represented by perfect fluid have studied by Coley and Tupper (1986) and Coley (1988). An interacting two fluid FRW universe is investigated by Pradhan et.al. (2011). Two fluid anisotropic cosmological models have been studied by Coley and Dunn (1990), Pant and Oli (2002), Oli (2008), Adhav et.al. (2011).

Manuscript revised on January 25, 2020; and published on February 10,2020

S. M. Botikar, Department of Applied Science, Sipna College of Engineering and Technology, Amravati, India. soniyanishant@gmail.com
In this paper we assume that the geometry of the universe is that of a Kantowski-Sachs space-time. The solution of field equations is obtained and physical behavior of the corresponding non-interacting fluids model is discussed.

\section{Metric ANd BaSiC Field Equations}

The Kantowski-Sachs space-time is given by $d s^{2}=d t^{2}-R^{2} d r^{2}-S^{2}\left(d \theta^{2}+\sin ^{2} \theta d \phi^{2}\right)$,

Where $R$ and $S$ are scale factors and are functions of time $t$ only.

The Einstein field equations are (For $8 \pi G=1, c=1$ ) given by

$R_{i}^{j}-\frac{1}{2} \delta_{i}^{j} R+\Lambda=-\left(T_{i}^{j(m)}+T_{i}^{j(r)}\right)$,

Where $R_{i}^{j}$ is the Ricci tensor, $R$ is the Ricci scalar and $T_{i}{ }^{j(m)}$ and $T_{i}{ }^{j(r)}$ are the energy momentum tensor for matter(perfect fluid) and radiation field respectively and $\Lambda$ is a cosmological constant.

The energy-momentum tensors for matter (perfect fluid) and radiation are given by

$$
\begin{aligned}
& T_{i}^{j(m)}=\left(\rho_{m}+p_{m}\right) u_{i} u^{j}-p_{m}, \\
& T_{i}^{j(r)}=4 / 3 \rho_{r} u_{i} u^{j}-1 / 3 \rho_{r}
\end{aligned}
$$

with components

$$
\begin{array}{cc}
T_{1}^{1(m)}=T_{2}^{2(m)}=T_{3}^{3(m)}=-p_{m} \quad, T_{0}^{0(m)}=\rho_{m} \\
T_{1}^{1(r)}=T_{2}^{2(r)}=T_{3}^{3(r)}=-\rho_{r} / 3 \quad, T_{0}^{0(r)}=\rho_{r}(4)
\end{array}
$$

where $\quad p_{m}, \rho_{m}$ and $\rho_{r}$ are pressure and densities of matter and radiation respectively.

Einstein's field equations (2) for line element (1) and with the equation (4) are 


\section{Available online at www.ijrat.org}

$2 \frac{\ddot{S}}{S}+\frac{\dot{S}^{2}}{S^{2}}+\frac{1}{S^{2}}+\Lambda=-p_{m}-\frac{\rho_{r}}{3}$

$\frac{\ddot{R}}{R}+\frac{\ddot{S}}{S}+\frac{\dot{R} \dot{S}}{R S}+\Lambda=-p_{m}-\frac{\rho_{r}}{3}$,

$$
\frac{2 \dot{R} \dot{S}}{R S}+\frac{\dot{S}^{2}}{S^{2}}+\frac{1}{S^{2}}-\Lambda=\rho_{m}+\rho_{r},
$$

Where overhead dots denotes derivatives with respect to cosmic time $t$.

The three independent equations (5) - (7) having five unknown $R, S, p_{m}, \rho_{m}, \rho_{r}$. Hence, to obtain the solution of these equations (5-7), we consider supplementary condition that the shear scalar is proportional to s scalar of expansion hear scalar, which yields the relation between metric potentials as

$R=A S^{n}$

Where $A$ and $n$ both are constants.

The equation of state $(\mathrm{EoS})$ of matter is given by

$$
p_{m}=(\gamma-1) \rho_{m}, \quad 1 \leq \gamma<4 / 3
$$

Using (8) and (9), the field equtions (5) - (7) reduces to $2 \frac{\ddot{S}}{S}+\frac{\dot{S}^{2}}{S^{2}}+\frac{1}{S^{2}}+\Lambda=-(\gamma-1) \rho_{m}-\frac{\rho_{r}}{3}$,

$(n+1) \frac{\ddot{S}}{S}+n^{2} \frac{\dot{S}^{2}}{S^{2}}+\Lambda=-(\gamma-1) \rho_{m}-\frac{\rho_{r}}{3}$,

$(2 n+1) \frac{\dot{S}^{2}}{S^{2}}+\frac{1}{S^{2}}-\Lambda=\rho_{m}+\rho_{r}$.

Subtracting equation (11) from (10), we get

$\ddot{S}+(n+1) \frac{\dot{S}^{2}}{S}+\frac{1}{1-n} \frac{1}{S}=0$

To solve equation (13), we denote $\dot{S}=\eta$ and $\ddot{S}=\eta \frac{d \eta}{d s}$.

Using above substitutions (13) reduces to

$$
\eta \frac{d \eta}{d s}+(1+n) \frac{\eta^{2}}{S}+\frac{1}{1-n} \frac{1}{S}=0
$$

Solving equation (14), we obtain

$$
\eta=\left[\frac{1}{n^{2}-1}+c S^{-2(1+n)}\right]^{1 / 2},
$$

where $c$ is a integration constant .

Hence the line element (1) reduces to

$d s^{2}=\left[\frac{1}{n^{2}-1}+c S^{-2(1+n)}\right]^{-1} d s^{2}-A^{2} S^{2 n} d r^{2}-S^{2}\left(d \theta^{2}+\sin ^{2} \theta d \phi^{2}\right)$
Considering suitable coordinate transformation, the equation (16) becomes

$d s^{2}=\left[\frac{1}{n^{2}-1}+c T^{-2(1+n)}\right]^{-1} d T^{2}-A^{2} T^{2 n} d r^{2}-T^{2}\left(d \theta^{2}+\sin ^{2} \theta d \phi^{2}\right)$

The geometrical and physical parameters for the above model are obtained. The expression for densities of matter $\rho_{m}$ and radiation $\rho_{r}$, the scalar of expansion $\theta$, the shear scalar $\sigma^{2}$, the deceleration parameter $q$ and spatial volume $V$ anisotropy parameter of expansion are given by

$$
\begin{aligned}
& \rho_{m}=\frac{1}{4-3 \gamma}\left[\frac{-2 c(1+2 n)}{T^{2(n+2)}}+\frac{2\left(n^{2}+n+1\right)}{\left(n^{2}-1\right) T^{2}}\right]+2 \Lambda \\
& \rho_{r}=\frac{3 c(2-\gamma)(2 n+1)}{(4-3 \gamma) T^{2(n+2)}}+\frac{6 n(1-\gamma)+n^{2}(2-3 \gamma)-2}{(4-3 \gamma)\left(n^{2}-1\right) T^{2}}+\Lambda \\
& p_{m}=\frac{\gamma-1}{(4-3 \gamma)}\left[\frac{2\left(n^{2}+n+1\right)}{\left(n^{2}-1\right) T^{2}}-\frac{2 c(1+2 n)}{T^{2(n+2)}}\right] \text {, } \\
& \theta=\frac{n+2}{T}\left[\frac{1}{n^{2}-1}+c T^{-2(1+n)}\right]^{1 / 2}, \\
& \sigma^{2}=\frac{2(n-1)}{3(1+n) T^{2}}+\frac{2 c(1-n)^{2}}{3 T^{2(2+n)}} \text {, } \\
& q=\frac{3 c(n+1) T^{-2(1+n)}}{(n+2)\left[\frac{1}{n^{2}-1}+c T^{-2(1+n)}\right]}-\frac{n-1}{n+2} ; n \neq-2 \\
& V=A T^{n+2} \text {, } \\
& \Delta=\frac{1}{3} \sum_{i=1}^{3}\left(\frac{H_{i}-H}{H}\right)^{2}=2\left(\frac{n-1}{n+2}\right)^{2} .
\end{aligned}
$$

\section{DISCUSSION}

It is observed that both the energy densities for matter and radiation are positive, provided $n>1$. The densities $\left(\rho_{m}\right.$ and $\rho_{r}$ ) and the scalar of expansion ( $\theta$ ) diverges as $T \rightarrow 0$. Whereas $\rho_{m}$ and $\rho_{r}$ converges to finite when $T \rightarrow \infty$. Hence the model has initial Big-Bang type singularity and represents the shearing and non-rotating expanding universe. For $n=1$, the shear scalar $\sigma$ is zero. Therefore for $n=1$ is the isotropizes. The spatial volume $V \rightarrow 0$ as $T \rightarrow 0$ and $V \rightarrow \infty$ as $T \rightarrow \infty$.i.e. universe starts with zero volume and increases with increase in time 
$T$ for $n>0$. The model accelerate or decelerate depends on the particular value of $n$. The deceleration parameter is zero for $n=1$ which indicates the universe is expanding with uniform velocity. It is observed that model does not exists for $n=1$. The value of the anisotropic parameter of the expansion is constant that means the anisotropy is maintained throughout the end of universe.

\section{SPECIFIC MODELS}

\begin{tabular}{|c|c|c|c|}
\hline Parameter & $\begin{array}{c}n=0 \\
\Lambda=-1\end{array}$ & $\begin{aligned} n & =2 \\
\Lambda & =-1\end{aligned}$ & $\begin{array}{l}n=-3 \\
\Lambda=-1\end{array}$ \\
\hline$\rho_{m}$ & $\frac{2}{3 \gamma-4}\left[\frac{c}{T^{4}}+\frac{1}{T^{2}}\right]-2$ & $\frac{1}{4-3 \gamma}\left[\frac{14}{3 T^{2}}-\frac{10 c}{T^{8}}\right]-2$ & $\frac{1}{4-3 \gamma}\left[\frac{10 c}{T^{-2}}+\frac{14}{8 T^{2}}\right]-2$ \\
\hline$\rho_{r}$ & $\frac{3 c(2-\gamma)+2 T^{2}}{(4-3 \gamma) T^{4}}-1$ & $\frac{45 c(2-\gamma)+(18-24 \gamma) T^{6}}{(4-3 \gamma) 3 T^{8}}-1$ & $\frac{-120 c(2-\gamma) T^{4}-9 \gamma-2}{(4-3 \gamma) 8 T^{2}}-1$ \\
\hline$\theta$ & $\frac{2}{T}\left[c T^{-2}-1\right]^{1 / 2}$ & $\frac{4}{T}\left[c T^{-6}-1\right]^{1 / 2}$ & $\frac{-1}{8 T}\left[8 c T^{4}+1\right]^{1 / 2}$ \\
\hline$\sigma^{2}$ & $\frac{2\left(c-T^{2}\right)}{3 T^{4}}$ & $\frac{2\left(1+3 c T^{-6}\right)}{9 T^{2}}$ & $\frac{4\left(1+8 c T^{4}\right)}{3 T^{2}}$ \\
\hline$V$ & $A T^{2}$ & $A T^{4}$ & $A T^{-1}$ \\
\hline$q$ & $\frac{3 c T^{-2}}{2\left[c T^{-2}-1\right]}+\frac{1}{2}$ & $\frac{9 c}{4 T^{6}}+2$ & $\frac{6 c T^{4}}{1 / 8+c T^{4}}-4$ \\
\hline$\Delta$ & $\frac{1}{2}$ & $\frac{1}{8}$ & 32 \\
\hline
\end{tabular}

In the above table we have given matter density, radiation density, the scalar of expansion, spatial volume, the shear scalar, the deceleration parameter and the anisotropic parameter of the expansion for different values of $n$. The comparative study of above models shows that $n=2$ gives satisfactory results for $c=1$ and $1 \leq \gamma<4 / 3$. More precise value of $\Lambda$ from recent observations will give standard model of universe.

\section{CONFLICT OF INTEREST}

On behalf of author states that there is no conflict of interest.

\section{REFERENCES}

[1] Adhav et. al.: Cont.Eur.J.Phys. 9(4), 919-925 (2011).

[2] Adhav et.al.: EJTP ,25,1-8 (2011).

[3] Coley A.A., Dunn K.: Astrophys.J.348, 26(1990).

[4] Coley A.A., Tupper B.O.J.: J.Math. Phys. 27,406(1986)

[5] Coley A.A.: Astrophys. Space Sci.140, 175 (1988).

[6] Davidson W.: Mon. Not. R. Astron. Soc. 124, 79 (1962).

[7] Gasprini M.,et.al.: Phys Rept.373,1-212 (2003).

[8] Gron : Journal of Mathe. Phys. 32, 2456 (1986).

[9] Knop et.al.: The Astrophysical J., 598,102 (2003).

[10] Krori et.al.: Journal of Mathe. Phys., 36,991-1553 (1995).

[11] Li , Hao: arXiv:astro-ph/0308288 (2003).
[12] Lorenz D.: Astrophysics and Space Sci, 98, 101-113 (1984).

[13] Matravers D.R.: Gen Rel. and Gravitation, 20,279 (1988).

[14] McIntosh, C.B.G.:Mon. Not. R. Astron.Soc.140,461(1968).

[15] Pant D.N.,Oli S.: Astrophys, Space Sci.281,623(2002).

[16] Pant D.N.,Oli S.: Astrophys, Space Sci.281,623(2002).

[17] Pradhan A., Amirhashchi H. \& Saha B. : Astrophys.Space Sci. 33,1,(2011).

[18] Pradhan and Yadav: Int. J. Mod. Phys. D 11, 893 (2002).

[19] Riess A.G.:et al.: Astophys. J.607, 665 (2004).

[20] Roy Choudhari, A.K.: Theoretical cosmology, Oxford, Clarendon Press (1979).

[21] Sanjay Oli : Astrophys Space Sci 314, 89-94 (2008).

[22] Sanjay Oli : Astrophys Space Sci 314, 95-103 (2008).

[23] Sing T., Agrawal A.: Astrophys Space Sci., 182, 289-312 (1991).

[24] Weber: Journal of Mathe. Phys. 25, 3279 (1984).

[25] Weber : Journal of Mathe. Phys. 26, 1308 (1985).

\section{AUTHORS PROFILE}

Dr. S. M. Borikar completed her Ph.D.in Mathematics from Sant Gadge

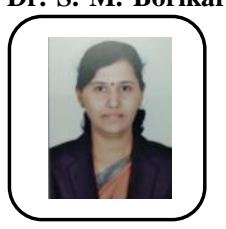
Baba Amravati University, Amravati. Currently working as Assistant Professor at Sipna College of Engineering and Technology, Amravati. She has presented papers at various National and internationa conferences both home and abroad, published articles and papers in various international journals. Her main area of interest is in relativity. 